\title{
Self-Organized Ordering of Nanostructures Produced by Ion-Beam Sputtering
}

\author{
Mario Castro, ${ }^{1}$ Rodolfo Cuerno, ${ }^{2}$ Luis Vázquez, ${ }^{3}$ and Raúl Gago ${ }^{4}$ \\ ${ }^{1}$ Grupo Interdisciplinar de Sistemas Complejos (GISC) and Grupo de Dinámica No Lineal (DNL), \\ Escuela Técnica Superior de Ingeniería (ICAI), Universidad Pontificia Comillas, E-28015 Madrid, Spain \\ ${ }^{2}$ Departamento de Matemáticas and Grupo Interdisciplinar de Sistemas Complejos (GISC), \\ Universidad Carlos III de Madrid, Avenida de la Universidad 30, E-28911 Leganés, Spain \\ ${ }^{3}$ Instituto de Ciencia de Materiales de Madrid, CSIC, Cantoblanco, E-28049 Madrid, Spain \\ ${ }^{4}$ Centro de Microanálisis de Materiales, Universidad Autónoma de Madrid, Cantoblanco, E-28049 Madrid, Spain
}

(Dated: February 2, 2008)

\begin{abstract}
We study the self-organized ordering of nanostructures produced by ion-beam sputtering (IBS) of targets amorphizing under irradiation. By introducing a model akin to models of pattern formation in aeolian sand dunes, we extend consistently the current continuum theory of erosion by IBS. We obtain new non-linear effects responsible for the in-plane ordering of the structures, whose strength correlates with the degree of ordering found in experiments. Our results highlight the importance of redeposition and surface viscous flow to this nanopattern formation process.
\end{abstract}

PACS numbers: $79.20 . \mathrm{Rf}, 68.35 . \mathrm{Ct}$, 81.16.Rf, 05.45.-a

Performance of many of the (opto)electronic devices currently being designed based on arrays of nanostructures such as quantum dots, requires a high degree of in-plane ordering [1]. Currently, there is a formidable effort to develop experimental techniques which are able to provide highly ordered nanostructures in a self-organized fashion [2]. These would allow for easy, low-cost and large area fabrication of patterned structures. Among these techniques, erosion by ion beam sputtering (IBS) at low energies [3] is a promising candidate [4, 5, 6], leading to production of nanostructures of varying degree of uniformity and order, onto diverse substrates such as GaSb, InP, and Si. Therefore, detailed knowledge of the basic mechanisms underlying erosion by IBS is crucial to understand and control the associated manufacturing process. From a fundamental point of view, the dynamics of surfaces eroded by IBS exemplifies neatly the interplay of fluctuations, external driving and dynamic instabilities, sharing many features with seemingly unrelated non-equilibrium systems, such as aeolian sand dunes [7, 8]. Thus, typically surfaces eroded by IBS spontaneously develop submicrometric patterns (dots, pits, ripples) 9] depending on experimental conditions, may deteriorate and eventually lead to rough interfaces, with fluctuations described by the universality classes of kinetic roughening [10].

A successful approach to surface erosion by IBS is provided by continuum evolution equations for the surface height, allowing access to time and length scales typical of the corresponding pattern formation process. This approach was pioneered by Bradley and Harper $(\mathrm{BH})$ [1], who, based on Sigmund's linear cascade approximation of sputtering in amorphous or polycrystalline targets [3], derived a linear equation that describes satisfactorily the main features of ripple formation under IBS, such as their alignment with the ion beam as a function of incidence angle. Additional features, such as ripple stabilization, wavelength dependence with ion energy or flux, or production of dot or hole structures as a function of bombardment conditions, required extensions of BH's approach 12, 13], leading to a non-linear equation of the Kuramoto-Sivashinsky (KS) type. The KS equation provides the continuum description of interfaces appearing in many diverse systems, see in [12], in which a periodic pattern develops, with a preferred wavelength (i.e. the lateral size of the nanostructures), that evolves into a disordered array. Thus, the crucial properties of homogeneity and in-plane short-range hexagonal ordering of the nanostructures produced by IBS remain to be understood [14]. Recent attempts have been made at extending the KS equation to overcome such shortcomings [15, 16], that do not provide definitive answers, since they either conflict with symmetries of the physical system, or with mathematical requirements for well-posedness [17].

In this Letter, we present a new continuum model of erosion by IBS. It leads to a physically and mathematically well defined generalization of the KS equation that explains within an unified framework the varying degree of homogeneity and order of the nanostructure arrays produced [4, [5, [6], as a function of experimental parameters. We exploit connections with ripple formation in sand dunes [7], hinted at by Aste and Valbusa 18], overcoming limitations of previous theories [1, 12, 13, 14, 15, 16].

Model.- During IBS, the bombarding ions penetrate the target and induce complex collision cascades in the bulk. In semiconductor substrates like those studied in [4, 5, 6], these cascades amorphize the near-surface layer. Sputtering events take place when surface atoms receive enough energy and momentum to break their bonds and leave the target. We will assume that only a fraction of those atoms are redeposited at the surface. Adatoms are moreover available to relaxation mechanisms such as surface diffusion, that can be thermally activated, or else 
be induced by the mentioned change in the local viscosity of the material close to the surface [19].

In the spirit of the so-called hydrodynamic theory of ripples in aeolian sand dunes [7], we define two coupled fields, namely, $R(\mathbf{x}, t)$ and $h(\mathbf{x}, t)$, where $\mathbf{x}=(x, y)$. The first one represents the fraction of surface atoms that are not sputtered away but, rather, remain mobile along the target surface. Analogously, $h$ measures the height of the surface neglecting the contribution from the fraction of mobile atoms $R$. Time evolutions of $R$ and $h$ are coupled through reaction and transport mechanisms [18]. Thus,

$$
\begin{aligned}
\partial_{t} h & =-\Gamma_{e x}+\Gamma_{a d}, \\
\partial_{t} R & =(1-\phi) \Gamma_{e x}-\Gamma_{a d}-\mathbf{v} \cdot \nabla R-\nabla \cdot \mathbf{J},
\end{aligned}
$$

where $\Gamma_{e x}$ and $\Gamma_{a d}$ are, respectively, the rates of excavation and addition to the surface, $\mathbf{v}$ is the average velocity of mobile atom, and $\phi \neq 0$ is the fraction of adatoms that detach irreversibly from the surface. Thus, system (10)-(2) does not conserve the amount of material, in marked contrast with typical conditions for aeolian sand dunes [8]. Here, large redeposition of sputtered atoms corresponds to the small $\phi$ limit, while, in the absence of redeposition, $\phi=1$. Considering that matter transport along the surface is due to diffusion of mobile species, we set $-\nabla \cdot \mathbf{J}=D \nabla^{2} R$, where $D$ is the surface diffusivity.

In the absence of bombardment, the concentration of mobile adatoms $R$ changes due to thermal nucleation of adatoms from the "immobile state" $h$, and subsequent transport along the surface. Assuming nucleation events are more likely in surface protrusions, we have $\Gamma_{a d}^{\text {no er. }}=\tau^{-1}\left[R-R_{e q}^{0}(1+\Lambda \kappa)\right]$, analogous of the GibbsThompson relation, $\kappa$ being the mean surface curvature and $\Lambda$ the capillary length, assumed isotropic due to amorphization by the ion beam. Here $\tau$ is related to the mean time between nucleation events, and $R_{e q}^{0}$ is the mean equilibrium concentration of mobile species for a flat surface. In the presence of bombardment, $\Gamma_{a d}^{\text {no er. }}$ has to be generalized, to include the contribution of erosion to surface mobility [19]. If the ions fall onto the target along the $x$ direction, forming angle $\theta$ with the normal to the uneroded target, we have, for small slopes [20, 21],

$$
\begin{aligned}
\Gamma_{e x} & =\alpha_{0}\left[1+\mu_{2}(\nabla h)^{2}\right]\left(1+\boldsymbol{\alpha}_{1} \cdot \nabla h+\alpha_{2} \nabla^{2} h\right) \\
& -\alpha_{0}\left[\alpha_{3}(\nabla h)^{2}-\alpha_{4}\left(\partial_{x} h\right)\left(\nabla^{2} h\right)\right]-\beta \nabla^{2} h, \\
\Gamma_{a d} & =\gamma_{0}\left[R-R_{e q}\left(1-\gamma_{2} \nabla^{2} h\right)\right],
\end{aligned}
$$

where $R_{e q}$ and $\gamma_{i}$ generalize parameters in $\Gamma_{a d}^{\text {no er. }}$ so that $\gamma_{0}=\tau^{-1}+\tau_{e x}^{-1}, \gamma_{2}=\Lambda+\Lambda_{e x}$, with $\tau_{e x}^{-1}$ and $\Lambda_{e x}$ being analogs of nucleation time and capillary length of erosive origin [7, 19]. Coefficients $\alpha_{i} \geq 0$ in (3) are related to geometric correction factors that take into account the local variation of the ion flux with the surface slopes [20]. E.g., for oblique incidence, $\alpha_{1}, \alpha_{4} \propto \sin \theta$, and $\alpha_{3}=1 / 2$. Likewise, coefficient $\mu_{2} \geq 0$ is related to the local variation of the sputtering yield with the surface slope 22], assumed to have a local minimum for normal incidence, while $\beta \geq 0$ measures the efficiency of erosion due to direct impingement of the ions onto surface atoms (knock-on sputtering) 3, 9]. The positive sign of $\alpha_{2}$ implements the physical instability inherent to Sigmund's theory, by which erosion is more efficient at surface depressions than at surface protrusions [3]. Actually, the analysis presented below will allow us to relate some of these coefficients with the parameters characterizing Sigmund's distribution of energy deposition.

Surface dynamics.- Our continuum model of IBS, formed by Eqs. (11)-(2), (3)-(4), provides a way to introduce systematically all relevant physical mechanisms for IBS, differing from that in [18] in a number of features. Rather than considering its full solution, we proceed by deriving an effective equation for the surface height. As in the experiments of references [4, 5, 6], we consider the case of ions bombarding the target at normal incidence $(\theta=0)$, thus $\alpha_{1}=\alpha_{4}=|\mathbf{v}|=0$ in (2), (3) [23]. After a transient time of order $\gamma_{0}^{-1}$, Eqs. (11)-(2) have a planar solution $h_{0}(t)=-\alpha_{0} \phi t, R_{0}(t)=R_{e q}+(1-$ ф) $\alpha_{0} / \gamma_{0}$. Perturbing this solution with periodic waves of the form $h_{k}=\tilde{h}_{k} \exp \left(\omega_{k} t+\mathrm{ik} \cdot \mathbf{x}\right)$, and an analogous expression for $R_{k}$, amplification/decay of such perturbations is characterized by the dispersion relation $\omega_{k}=$ $R_{e q} \gamma_{0} \gamma_{2}\left(\epsilon \phi k^{2}-\gamma_{0}^{-1}(D+\phi A)[1-\epsilon(1-\phi)] k^{4}\right)$, with $\epsilon=$ $A /\left(R_{e q} \gamma_{0} \gamma_{2}\right)$ and $A=\alpha_{0} \alpha_{2}-\beta$. If $A>0$ in $\omega_{k}$, i.e. if sputtering is dominated by collision cascades rather than knock-on events, as occurs at low to intermediate energies where Sigmund's theory is applicable, there is a band of unstable modes that grow exponentially fast, with a linear dispersion relation $\omega_{k}$ of the expected KS type. At this stage, the surface morphology is dominated by a periodic pattern whose wave-vector maximizes $\omega_{k}$. In-plane isotropy under normal incidence implies dependence of $\omega_{k}$ on $k=|\mathbf{k}|$ rather than the full wave-vector $\mathbf{k}$, thus the surface power spectral density is, rather, maximum on a ring [6, 14]. Stabilization of this pattern occurs when its amplitude is large enough that non-linear effects are no longer negligible. Close to the instability threshold, the rate of erosion is much smaller than the rate of addition to the surface. Hence, parameter $\epsilon$ above, which is the ratio between these two typical rates, is small. We thus can perform a multiple scale expansion by introducing time scales $T_{1}=\epsilon t$ and $T_{2}=\epsilon^{2} t$, and by rescaling length scales as $X=\epsilon^{1 / 2} x$. To lowest non-linear order $\mathcal{O}(\epsilon)$ and as seen in the slow variables $\left(X, T=T_{1}+T_{2}\right)$, surface dynamics is described by (see [20] for details) 24]

$$
\left.\partial_{T} H=-\nu \nabla^{2} H-\mathcal{K} \nabla^{4} H+\lambda_{1}(\nabla H)^{2}-\lambda_{2} \nabla^{2}(\nabla H){ }^{2} 5\right)
$$

where $H=h_{1}+\epsilon h_{2}$, and

$$
\begin{aligned}
\nu & =A \phi, \quad \mathcal{K}=\epsilon \gamma_{0}^{-1}(D+\phi A)\left[R_{e q} \gamma_{0} \gamma_{2}-A(1-\phi)\right], \\
\lambda_{1} & =\phi \alpha_{0}\left(1 / 2-\mu_{2}\right), \\
\lambda_{2} & =\epsilon \alpha_{0}\left(1 / 2-\mu_{2}\right) \gamma_{0}^{-1}\left[(D+\phi A)(1-\phi)-R_{e q} \phi \gamma_{0} \gamma_{2}\right] .
\end{aligned}
$$


Eq. (5) with a noise term, has been already employed in the growth of amorphous thin films 25]. In our context, Eq. (5) has some important limits. First, in the absence of ion bombardment, $A=\alpha_{0}=0, \gamma_{0} \rightarrow \tau^{-1}$ and $\gamma_{2} \rightarrow \Lambda$, and in the original variables (5) reduces to Mullins' equation for thermal surface diffusion [26], $\partial_{t} h=-D R_{e q} \Lambda \nabla^{4} h$. In the general case, (5)-(6) include contributions to surface diffusion that are both thermally activated, and directly induced by the ion beam as in 19]. Second, the BH limit corresponds to $\phi=1$, i.e., no redeposition. While in [18] the $\mathrm{BH}$ limit zeroes out the $k^{4}$ contribution to the analog of $\omega_{k}$-thus making the typical length scale of the dot structures remain undefined within linear instability - , here Eq. (51) recovers for $\phi=1$ the equation obtained within BH's approach to Sigmund's theory 12, 16], including the fact that the coefficients of the two nonlinear terms have the same signs thus making the equation nonlinearly unstable and mathematically ill-posed 16, 17]. Thus, beyond its experimental relevance, redeposition is crucial in order to make the theory mathematically sound. On the other hand, the $\mathrm{BH}$ limit allows us to extract the phenomenological dependence of the parameters in our model with characteristics of the collision cascades, such as the ion penetration depth, $a$, and the longitudinal and lateral widths $\sigma$, and $\mu$, characterizing the Gaussian decay of enery deposition 3]. Thus, for $\phi \rightarrow 1$ we have, in the notation of [12], $\alpha_{0}=F, \alpha_{2}=a \mu^{2} /\left(2 \sigma^{2}\right), \mu_{2}=1-\mu^{2} /\left(2 \sigma^{2}\right)-\mu^{2} /\left(2 \sigma^{4}\right)$, $R_{e q} \gamma_{2}=\mu^{2} / 4$, with $F \propto J E / \sigma$, where $J$ and $E$ are the average ion flux and energy, respectively.

Eq. (5) describes the evolution of the erosion process. Initially, dynamics is controlled by the linear terms, with the same dispersion relation $\omega_{k}$ as above, and a periodic pattern develops, with characteristic wavelength given by

$$
l_{c}=2 \pi\left[2 R_{e q} \gamma_{2}(D+\phi A)[1-\epsilon(1-\phi)] /(A \phi)\right]^{1 / 2},
$$

providing the typical size of the nanostructures that form. When local slopes become large, the nonlinear terms in Eq. (5D) control the dynamics in an opposing way. While the $\lambda_{2}$ term tends to coarsen the nanostructures in amplitude and lateral size, similarly to its rôle in the coarsening of ripples on aeolian sand dunes [7], the nonlinearity $\lambda_{1}$ tends to disorder the pattern leading to the paradigmatic KS spatiotemporal chaos. Remarkably, $\lambda_{1}(\nabla h)^{2}$ seems to interrupt the coarsening process induced by $-\lambda_{2} \nabla^{2}(\nabla h)^{2}$ and the stationary state morphology consists of domains of hexagonally ordered nanostructures separated by defects. The density of these is a function of the ratio $r=\lambda_{2} / \lambda_{1}$, whose $r \rightarrow 0$ limit in Eq. (5) leaves us with the KS equation. In Fig. 1 $(a)$, we plot the stationary-state morphology obtained by numerical integration of Eq. (5) for a relatively large ratio $r=5$ [27]. The high degree of in-plane short range hexagonal ordering is made clear by the height autocorrelation function, shown in the inset of Fig. 目 $(a)$. The time evolution of the dot pattern can be assessed in Fig. 2 $(a)$, in which the surface roughness (mean height square deviation) $W(t)$ vs $t$ is shown for the same parameters as in Fig. 1 $(a)$. In excellent agreement with measurements for nanodots on GaSb [14], the roughness first increases exponentially during development of the linear instability, attains a maximum value after dots have coarsened to form a densely packed array, and finally relaxes to a smaller stationary value when defects among different dot domains are annihilated. Times between linear instability and maximum in the roughness correspond to non-linear coarsening of the dot structures, as seen in the plot of the lateral correlation length $\xi_{c}(t)$, shown on the same panel. We define $\xi_{c}(t)$ as the lengthscale provided by the first secondary maximum of the height autocorrelation. As seen in Fig. 2( $(a), \xi_{c}(t)$ scale is constant during linear instability, grows as $t^{0.27 \pm 0.02}$, and saturates at long times, in agreement with experiments on InP [5]. This interrupted coarsening process has been also observed on Si [6] (b) and GaSb [14].

Experimental conditions reflect in the value of $r$ [28], and can be such that this parameter is substantially smaller. Dynamics is then closer to that of the KS equation. The intermediate coarsening regime narrows, and is followed by kinetic roughening. A surface morphology produced in these conditions $[r=0.5]$ is shown in Fig. $1(b)$, which can be compared with an AFM scan $[(d)]$ of a Si target irradiated as in [6]. Again, agreement is excellent. Note that the morphology now differs appreciably from that of the KS equation, displayed in Fig. 1( $(c)$. While for Eq. (5) a short-range ordered pattern coexists with long-range disorder and roughening, in the pure KS system disorder of the cellular structure is paradigmatic, see the height autocorrelations in Figs. I $(b),(c)$. Still, the time evolution of the roughness in Fig. 2(b) (o), predicted by Eq. (51) for small $r$ values, is similar to that of the $\mathrm{KS}$ case, Fig. 2 $(b)(+)$ : initial rapid growth is followed by much slower dynamics, and saturation to the stationary state. Such is also the experimental behavior found for nanostructures produced on $\mathrm{Si}$, see Fig. 3 in [6] (a). Comparing the two plots in Fig. 2(b), for small (non-zero) $r$ values the small-scale nonlinearity $\lambda_{2}$ is seen to stabilize the linear instability earlier, and leads to smaller stationary roughness. Moreover, in contrast with Fig. 2(a), Fig. 2 (b) shows that for small or zero $r$ values, the roughness does not have a local maximum as a function of time.

In summary, we have introduced a continuum model for the formation of nanometric sized patterns by IBS. The model accounts within an unified framework for experimental features of nanopatterns recently produced on diverse materials. Moreover, it leads to an effective interface equation providing new predictions. Thus, considering dependencies [3] on ion energy $E$ of the features of the distribution of deposited energy, $a, \mu, \sigma$, the dot size $l_{c}$ behaves, in the large redeposition limit $\phi \lesssim 1$, as $l_{c} \sim[E+\text { const. }]^{1 / 2}$. For small $E$, this implies $l_{c}$ is energy independent, while $l_{c} \sim E^{1 / 2}$ for large enough energies. 
Observations exist [14, 29] compatible with such energy dependence, although a systematic study assessing the importance of redeposition would be highly desirable.

From a fundamental point of view, Eq. (5) also leads to new results. Specifically, this is a height equation with local interactions in which a pattern is stabilized with constant wavelength and amplitude, in contrast with conjectures for $1 d$ systems 30$]$. Although more theoretical work is still needed [e.g., regarding the asymptotic properties of Eq. (5)] this suggests that in $2 d$ patterns, coarsening dynamics is indeed more complex than in $1 d$ [31].

R. G. acknowledges a Ramón y Cajal Fellowship from MECD (Spain). This work has been partially supported by MECD (Spain) grants Nos. BFM2003-07749-C05, -05 (M. C.), -01 (R. C.), and -02 (L. V.).

[1] Springer Handbook of Nanotechnology, edited by B. Bhushan (Springer, Berlin, 2004).

[2] V. A. Shchukin and D. Bimberg, Rev. Mod. Phys. 71, 1125 (1999).

[3] P. Sigmund, in Sputtering by Particle Bombardment, edited by R. Behrisch (Springer-Verlag, Heidelberg, 1983), Vol. I, p. 9.

[4] S. Facsko et al., Science 285, 1551 (1999).

[5] F. Frost, A. Schindler, and F. Bigl, Phys. Rev. Lett. 85, 4116 (2000).

[6] R. Gago et al., Appl. Phys. Lett. 78, 3316 (2001); Nanotechnol. 13, 304 (2002).

[7] O. Terzidis, P. Claudin, and J.-P. Bouchaud, Eur. Phys. J. B 5, 245 (1998); A. Valance and F. Rioual, ibid. 10, 543 (1999); Z. Csahók, et al., Eur. Phys. J. E 3, 71 (2000).

[8] C. Misbah and A. Valance, Eur. Phys. J. E 12, 523 (2003).

[9] For reviews, see G. Carter, J. Phys. D: Appl. Phys. 34, R1 (2001); U. Valbusa, C. Boragno, and F. Buatier de Mongeot, J. Phys.: Condens. Matter 14, 8153 (2002).

[10] A.-L. Barabási and H. E. Stanley, Fractal Concepts in Surface Growth (Cambridge University Press, Cambdridge, 1995).

[11] R. Bradley and J. Harper, J. Vac. Sci. Technol. A 6, 2390 (1988).

[12] R. Cuerno and A.-L. Barabási, Phys. Rev. Lett. 74, 4746 (1995); M. Makeev, R. Cuerno, and A.-L. Barabási, Nucl. Instrum. Methods Phys. Res. B 197, 185 (2002).

[13] B. Kahng, H. Jeong, and A.-L. Barabási, Appl. Phys. Lett. 78, 805 (2001).

[14] T. Bobek et al., Phys. Rev. B 68, 085324 (2003).

[15] S. Facsko et al., Phys. Rev. B 69, 153412 (2004).

[16] T. C. Kim et al., Phys. Rev. Lett. 92, 246104 (2004).

[17] M. Castro and R. Cuerno, unpublished.

[18] T. Aste and U. Valbusa, Physica A 332, 548 (2004).

[19] C. C. Umbach et al., Phys. Rev. Lett. 87, 246104 (2001).

[20] M. Castro et al., in preparation.

[21] Due to their irrelevance at the $\theta=0$ condition considered here, we have ignored the anisotropies of the sputtering yield with substrate directions, studied in [20].

[22] M. Nastasi, J. W. Mayer, and J. K. Hirvonen, Ion-solid interactions: fundamentals and applications (Cambridge University Press, Cambridge, 1996). In [18], $\mu_{2}=0$.

[23] In [5] $\theta \neq 0$ is used, with simultaneous substrate rotation. Results below apply after azimuthal average [20].

[24] Actually, additional terms appear on the rhs of Eq. (5), namely, $\nabla \cdot(\nabla H)^{3}$, and $(\nabla H) \cdot \nabla^{3} H$. The former is cubic, rather than quadratic, in derivatives of $H$. The latter is multiplied by a small coefficient in the limit taken. Although these terms influence properties such as the dot shape [20], being small they do not change the qualitative properties of in-plane dot ordering of interest here.

[25] M. Raible et al., Europhys. Lett. 50, 61 (2000).

[26] W. W. Mullins, J. Appl. Phys. 28, 333 (1957).

[27] While $\lambda_{1}>0$ leads to dot structures, hole arrays ensue for $\lambda_{1}<0$ [13], and can be accounted here, in contrast with [18], for appropriate values of $\mu_{2}$. See experiments in, e.g., G. Costantini et al., Surf. Sci. 416, 245 (1998).

[28] As in the KS theory of IBS [see e.g. S. Facsko et al., Phys. Rev. B 63, 165329 (2001)], qualitative predictions are accurate, but quantitative predictions may deviate due to neglected mechanisms actually contributing to the interface equation with the same terms as considered.

[29] F. Frost et al., unpublished.

[30] J. Krug, Adv. Complex Systems 4, 353 (2001).

[31] P. Politi and C. Misbah, Phys. Rev. Lett. 92, 090601 (2004).
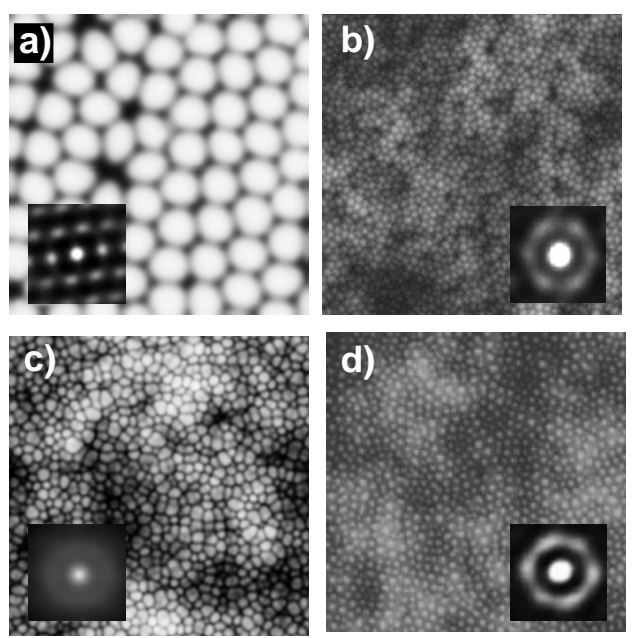

FIG. 1: (a) Stationary state morphology from the numerical solution of Eq. (5) with $\nu=2 \mathcal{K}=2, \lambda_{1}=0.1$, $\lambda_{2}=0.5$, lateral size $L=256$. Units are arbitrary. Inset: $2 d$-autocorrelation, showing high degree of short-range hexagonal order. (b) Same as before, for $\lambda_{1}=1, L=512$. (c) same as (b) for the KS equation, $\lambda_{2}=0$. (d) $3 \times 3 \mu \mathrm{m}^{2}$ AFM scan of a Si target irradiated as in Ref. [6] for $4 \mathrm{~h}$. 

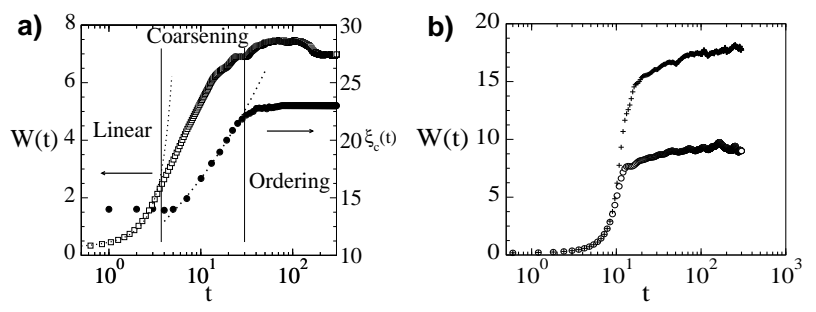

FIG. 2: $(a) W(t)$ and $\xi_{c}(t)$ from Eq. (5) for Fig. 1 $a$ ). Dotted line in the linear (coarsening) region grows as an exponential $\left(\right.$ as $\left.t^{0.27}\right)$. (b) $W(t)$ for Fig. M $(b)(\circ)$, and Fig. 1 $\left.c\right)(+)$. 